\title{
Tax Evasion Dynamics via Kinetic Models of Opinion Dynamics
}

\author{
F. W. S. Lima \\ Dietrich Stauffer Computational Physics Lab, Departamento de Física, Universidade Federal do Piauí, \\ Teresina, Brazil \\ Email: fwslima@gmail.com
}

How to cite this paper: Lima, F.W.S. (2019) Tax Evasion Dynamics via Kinetic Models of Opinion Dynamics. Theoretical Economics Letters, 9, 2354-2361.

https://doi.org/10.4236/tel.2019.97149

Received: August 20, 2019

Accepted: September 23, 2019

Published: September 26, 2019

Copyright (c) 2019 by author(s) and Scientific Research Publishing Inc.

This work is licensed under the Creative Commons Attribution International License $(\mathrm{CC}$ BY 4.0).

http://creativecommons.org/licenses/by/4.0/

\section{Open Access}

\begin{abstract}
The socioeconomic phenomena as tax evasion in agent-based community can be studied numerically through the Zaklan model (ZM). The ZM has been configured as an excellent tool to control the tax evasion fluctuations in communities with agents or individuals that can be considered tax evaders or honest. The dynamic of temporal evolution of the ZM was studied initially via the equilibrium Ising model (IM), non-equilibrium Majority-vote model (MVM) and recently via a three-state kinetic agent-based model. Here, through Monte Carlo simulations, we study the problem of the tax evasion fluctuations using a discrete version of the kinetic model of opinion dynamics (BCS) in the dynamic of the temporal evolution of the ZM. Unlike previous models cited here in the BCS model, the interactions between agents occur in pairs. Then, we found that the BCS model is as efficient as the IM and MVM in controlling the tax evasion fluctuations. This control is even better when we use strong punishment values $k$ even for low audit probabilities $p_{a}$.
\end{abstract}

\section{Keywords}

Opinion Dynamics, Sociophysics, Majority Vote, Non-Equilibrium

\section{Introduction}

The analysis of the social and economic behavior of a community of people fulfilled by Bloomquist (2006), Andreoni (1998), Lederman (2003), Slemrod et al. [1-6] (2003 to 2007), indicates that the tax evasion in a community is a major cause of concern for governments. Through empirical evidence Gächter [7] (2006), Frey and Torgler [8] (2006) have provided that the group members or neighborhood of tax evaders are important in deciding whether or not to pay taxes.

Zaklan et al. $[9,10]$ (2008) developed an economics model to study the problem of tax evasion dynamics on a people community using the equilibrium IM on a square lattice (SL) and Monte-Carlo simulations 
with the Glauber algorithms. In some complex networks like directed Barabási-Albert and Apollonian networks it is not possible to simulate the ZM via IM because this has no social noise on these complex networks, i.e., does not present a phase transition.

To solve this problem, Lima $[11,12](1992,2008)$ proposed the use of the non-equilibrium MVM to study the tax evasion in any complex network [13] (2010). He points out that people do not live in a social equilibrium, and any social noise can drive to a government or market chaos. Then, it is reasonable to expect that a non-equilibrium model (MVM) explains better events of non-equilibrium. In order to show that the problem of tax evasion fluctuations is better described by a non-equilibrium model than by an equilibrium model, Lima [14-17] (2010 to 2016) studied the ZM on Apollonian, Opinion-Dependent, Solomon, and Small-World Networks. In all these cases the tax evasion problem was analyzed using the two-state version of MVM, where the honest agent was rated +1 and the evaded value -1 .

Crokidakis [18] (2014) has studied the problem of tax evasion via ZM on a fully-connected population. In his work, the agents may be in three different states, namely honest taxpayers, tax evaders and undecided. The undecides agents are individuals in an intermediate class among honests and evaders. Each agent can change his state following a kinetic exchange opinion dynamics, where the agents interact by pairs with negative (probability $p$ ) and positive $(1-p)$ affinity parameters $\mu_{i j}$, representing agreement/disagreement between pairs of agents.

Biswas et al. [19] (2012) proposed a kinetic model of continuous opinion dynamics (BCS). The BCS model has mutual interactions that can be both positive and negative and a single parameter p representing the fraction of negative interactions. Numerical simulations of the continuous version of this model indicate the existence of a universal, continuous phase transition at $p=p_{c}$ with exponents of mean field $(\nu d=2.00(1), \beta=0.50(1)$ and $\gamma=1.00(1))$. Mukherjee and Chatterjee [20] studied the BCS model on square and cubic lattices (2D and $3 \mathrm{D})$. Their numerical results indicate that the critical behavior of the BCS model on these lattices is the same as that of the Ising model in the corresponding dimensions.

Here, we study the behavior of the tax evasion on an agents community of honest citizens and tax evaders, where the agents are positioned on sites of SL, but now using a discrete version of BCS model for the temporal evolution of ZM.

The article is organized as follows: we present, in the next section, the model and some of the Monte Carlo simulation details. In Section 3 the results are presented and, in the final section, some concluding remarks are discussed.

\section{ZM via Non-Equilibrium Dynamics of BCS Model}

We use the ZM via BCS model to study the tax evasion fluctuations on a community of homogeneous agents located on a SL. In every time period, each SL site is inhabited by an agent (individuals) with opinion $O_{i}$, who can either be an honest taxpayer $O_{i}=+1$ or a cheater $O_{i}=$ -1 . It is assumed that initially, everybody is honest. Each period the agents can rethink their behavior and have the opportunity to become the opposite type of agent they were in the previous period. Each 
agent's social SL may either prefer tax evasion or reject it. Individual decision making depends on four rules:

1) Each node in the SL is assigned a discrete opinion variable $O_{i}=$ \pm 1 ;

2) At each time step is randomly choose a network node or site to update;

3) Then one of your links is randomly selected, and a $\mu_{i j}$ parameter called binding affinity is set. The affinity parameter assumes values -1 with probability $p$ and +1 with probability $1-p$;

4) Both nodes $i$ and $j$ sharing the selected link will be updated according to the following expressions:

$$
\begin{aligned}
& O_{i}(t+1)=O_{i}(t)+\mu_{i j} O_{j}(t) \\
& O_{j}(t+1)=O_{j}(t)+\mu_{i j} 0_{i}(t),
\end{aligned}
$$

where $O_{i}(t)$ and $O_{j}(t)$ are the oldest states of opinion, and $O_{i}(t+1)$ and $O_{j}(t+1)$ are the updated states of opinion.

The ZM presents an enforcement mechanism that consists of two components: a probability of an efficient audit $p_{a}$; and a number $k$ of periods. Then once the tax evasion is detected the tax evaders, $O_{i}=-1$, can become honest individuals $O_{i}=+1$, in the presence of an audit probability $p_{a}$, for a number $k$ of periods. One time unit is one sweep through the entire system. Therefore, according to the above rules and unlike other models such as the IM or MVM, for example, agents' opinions are updated by pairs that were randomly connected by $\mu_{i j}$ affinity links. The ordering in the system is measured by the quantity namely average opinion defined by

$$
O=\left\langle\frac{1}{N}\left|\sum_{i=1}^{N} O_{i}\right|\right\rangle,
$$

where $<. . .>$ denotes configurational average taken at steady states.

The fraction of tax evaders is

$$
\text { tax evasion }=\left|\frac{N-N_{\text {honest }}}{N}\right|,
$$

where $N$ is the total number and $N_{\text {honest }}$ the honest number of agents. The tax evasion is calculated at every time step $t$ of system evolution. Here $N=10^{4}$ agents.

Similar to IM and MVM models in BCS model for $p>p_{c}$, half of the people are honest, $O_{i}=+1$, and the other half cheat $O_{i}=-1$, while for $p<p_{c}$ either one opinion or the other opinion dominates. Because of this behavior, we set a fixed noise $(p)$ to some values slightly below $p_{c}$, where the case that agents distribute in equal proportions onto the two alternatives is excluded. We set $p=0.95 p_{c}$ with $p_{c}=0.1340 \pm$ 0.0001 on SL. Then we vary the degrees of punishment $(k=10,50$ and $100)$ and audit probability rate $\left(p_{a}=0.5 \%, 10 \%\right.$ and $\left.90 \%\right)$. Therefore, if tax evasion is detected, the enforcement mechanism $p_{a}$ and the time of punishment $k$ are triggered in order to control the tax evasion level. The punished individuals remain honest for a certain number $k$ of periods, as explained before. 


\section{Results and Discussion}

Following the ZM, we start with a population of $N=10^{4}$ individuals where initially everyone is considered honest $O_{i}(t=0)=+1$. Then we apply the rules of the ZM as the punishment period $k$ and the audit probabilities $p_{a}$.

In Figure 1, we show the tax evasion as a function of time via a BCS model for two different values of $p>p_{c}=0.1340(1)$, namely $p=0.35$ Figure 1(a) and Figure 1(b) and $p=0.25$ ((c) and (d)). In this case, for $p>p_{c}$, we will have the baseline case $\left(k=0\right.$ and $\left.p_{a}=0\right)$, i.e., the kinetic exchange dynamics defined by Equation (1) and Equation (2) leads the system to a disordered state with an equal fraction $1 / 2$ (50\%) for \pm 1 states. Thus, from Figure 1 that if the audits are efficient $\left(p_{a}=90 \%\right)$ the tax evasion can be considerably reduced to $\approx 20 \%$ for $k=10$ and to $\approx 7.5 \%$ for $k=50$. This behavior of fluctuations of the tax evasion is identical to that reported in $\mathrm{ZM}$ on regular lattices and networks $[9,10,12,13]$. For the cases where $p_{a}=5 \%$, considered more realistic by the literature, the punishment is more efficient when the penalty duration is high as $k=50$. In this case, the tax evasion can be reduced for values around $30 \%$. Notice that when the value of $p$ decrease the fraction of tax evaders also decreases, that is, for high $p$ the fraction of opinions -1 is greater than in the cases of lower values of $p$. This behavior is due to the fact that there are many negative affinity parameters $\mu_{i j}$ in the population, which allows many transitions $O_{i}=+1 \rightarrow O_{i}=-1$ for $p>p_{c}$.

a

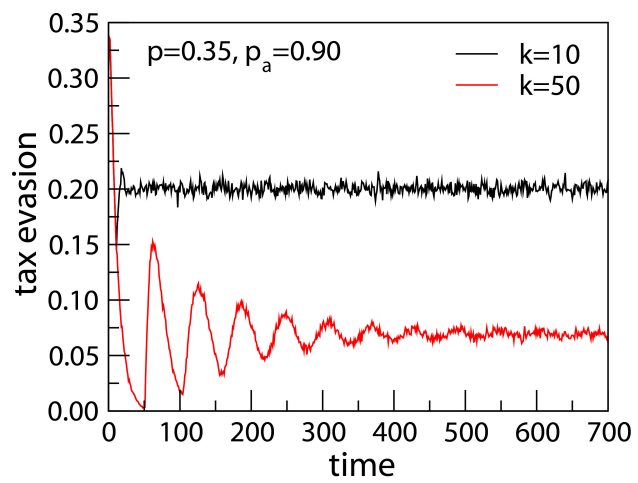

C

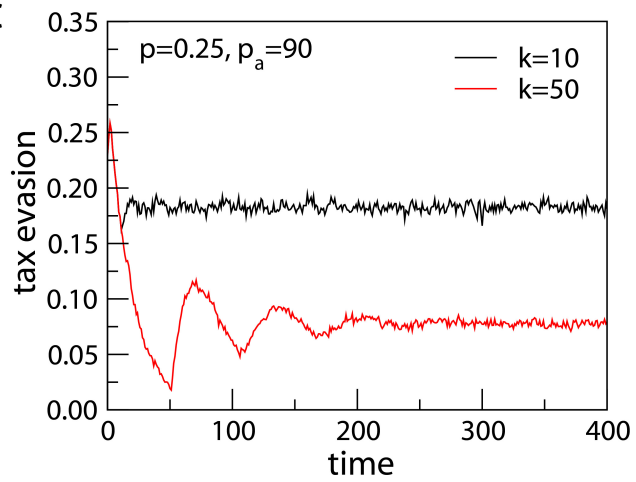

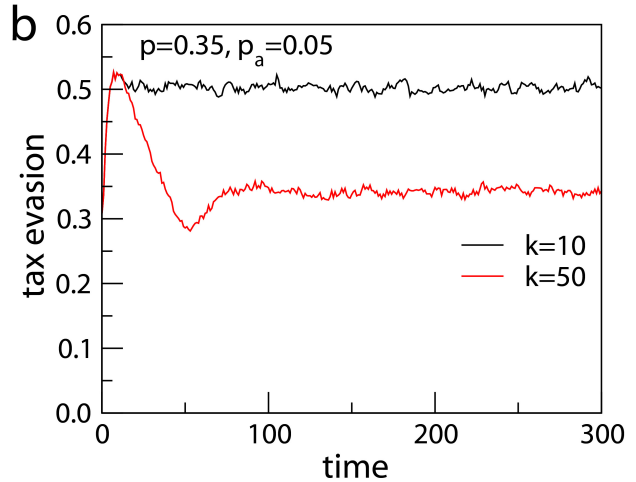

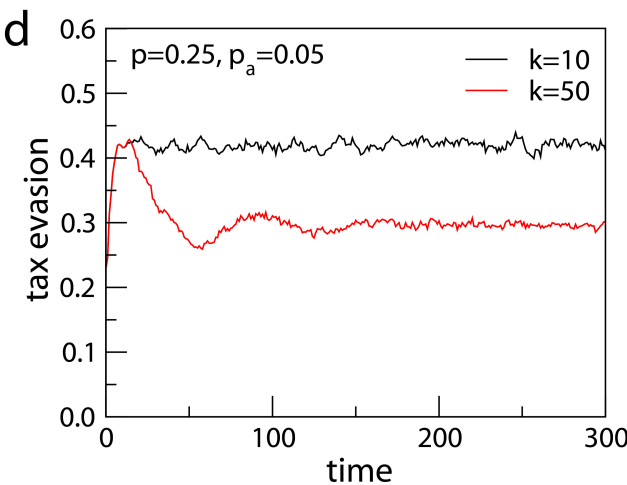

Figure 1. (Color online) Time evolution of tax evasion via BCS model for different values of degrees of punishment $k=10$ and 50 and two distinct audit probabilities $p_{a}=0.9$ ((a) and (c)) and $p_{a}=0.05((\mathrm{~b})$ and (d)). The results are for $p=0.35$ ((a) and (b)) and $p=0.25((\mathrm{c})$ and (d)). For realization of each curve, we use a population of size $N=10^{4}$ individuals at $p=0.95 p_{c}$. 
In Figure 2(a) and Figure 2(b), we present results for $p=0.15$, this is, another value of $p>p_{c}$ which is very close to the critical point $p_{c}$. See that for a high audit probability $p_{a}=90 \%$ the tax evasion can be dramatically reduced for both punishment periods $k=10$ and $k=50$. See also that even for $p_{a}=5 \%$ considered here as the realistic value in a community of tax evaders the application of severe punishments as $k=50$ can lead the evasion to low levels like $20 \%$, see Figure 2(b). Still, in Figure 2, we show results for $p<p_{c}$, Figure 2(c) and Figure $2(\mathrm{~d})$. Then for $p=0.05$ the time dynamics of the BCS model drive the system to a steady-state with a large number of honest individuals $\left(O_{i}=+1\right)$. For $p_{a}=90 \%$ (Figure $\left.2(\mathrm{c})\right)$ the tax evasion is reduced to $6 \%$ as $k=10$ and to $5 \%$ as $k=50$ and as $p_{a}=5 \%$ (Figure 2(d)) the tax evasion is now reduced to $6 \%$ regardless of the values of the punishment periods $k=10$ and 50 .

In Figure 3, we exhibit the average tax evasion in the stationary states as a function of the audit probability $p_{a}$. Figure 3(a)-(c) display the results for $p=0.05,0.15$ and 0.35 , respectively, and for punishment period values of $k=10,50$, and 100. For $p=0.05$, i.e., $p<p_{c}$ the average tax evasion is small enough and decreases with the growth of $p_{a}$. This decreases to $4.5 \%$ for high punishment period values like $k=100$. Otherwise, the tax evasion for $k=100$ and values of $p>p_{c}$ as 0.15 and 0.35 decrease for $6 \%$ and $5 \%$, respectively. In Figure $3(\mathrm{~d})$, we show the behavior of the tax evasion for $k=10$ and for $p=0.05$, 0.15 , and 0.35 . One can see that for $p<p_{c}$ the tax evasion remains constant for all values of $p_{a}$. For values of $p>p_{c}$ the tax evasion is reduced to $\approx 15 \%$ and $\approx 25 \%$ for 0.15 and 0.35 , respectively.

a
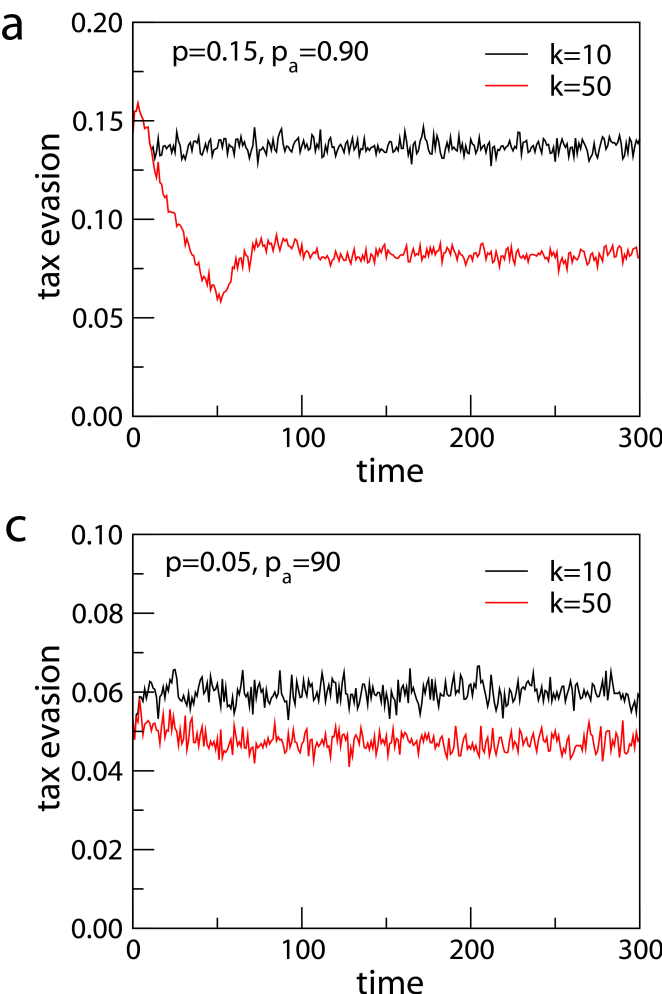
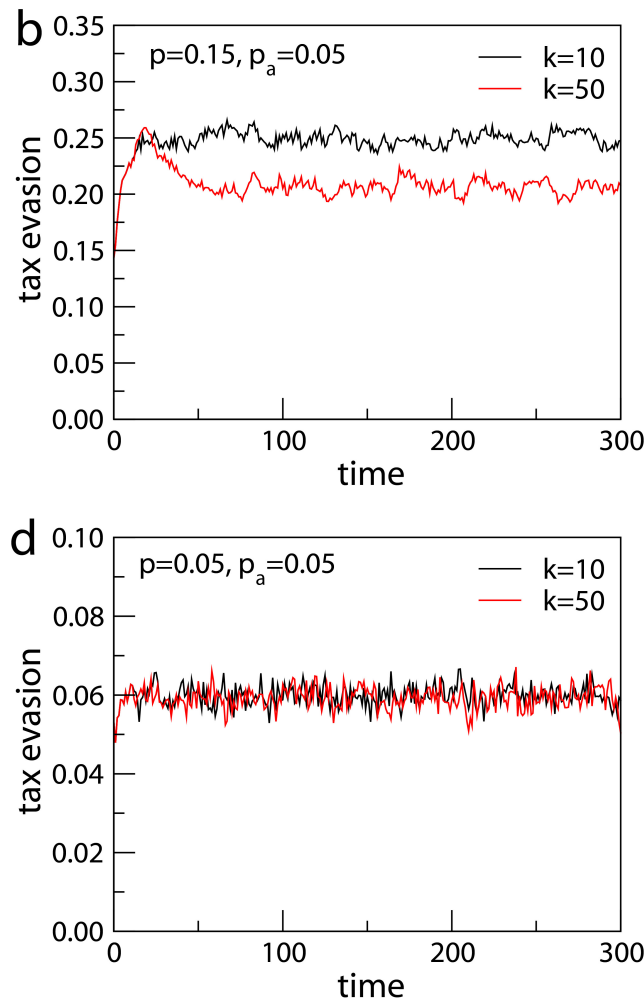

Figure 2. The same of Figure 1, but now to $p=0.15$ ((a) and (b)), and $p=0.05((\mathrm{c})$ and (d)). 

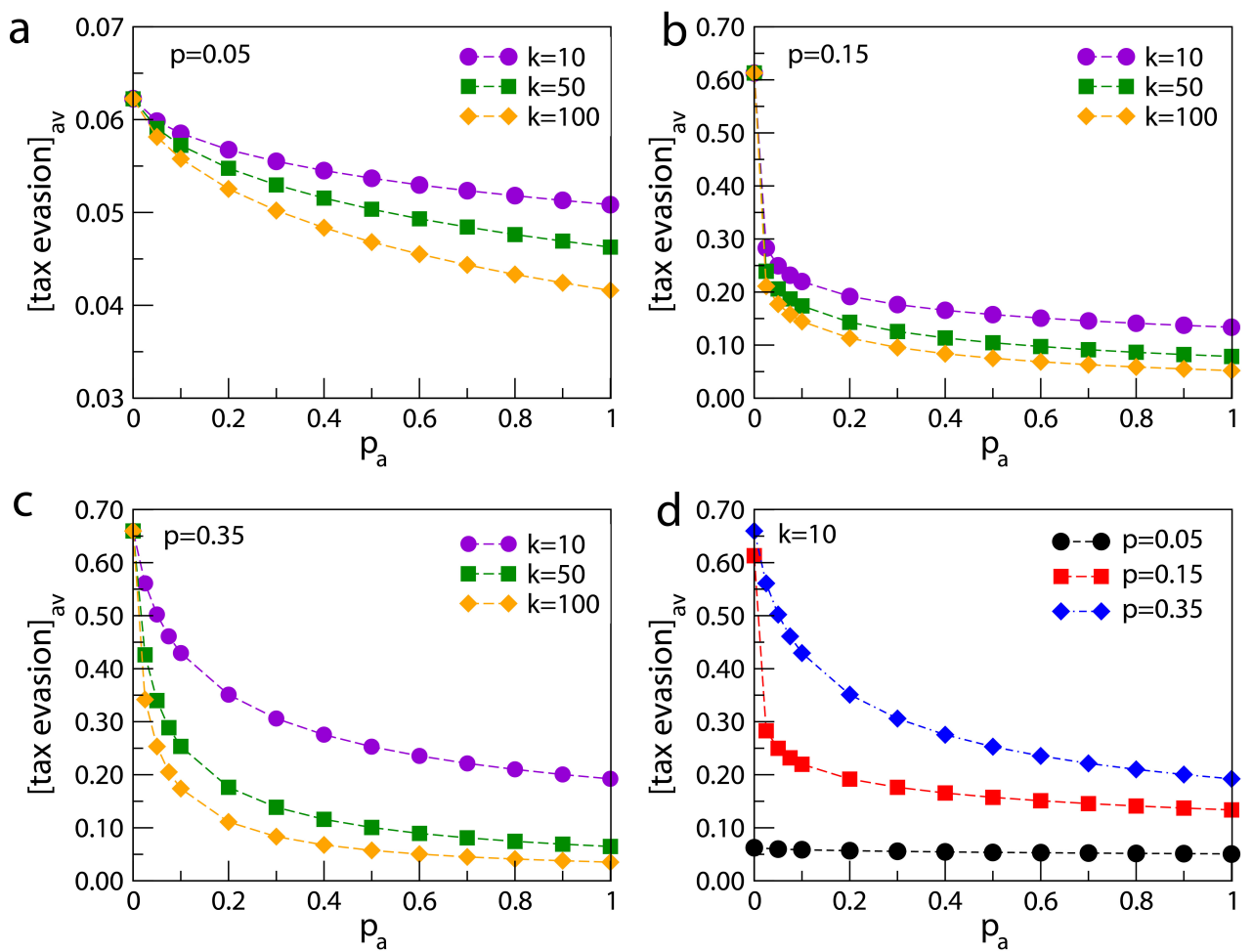

Figure 3. (Color online) Average stationary tax evasion as a function of the audit probability $p_{a}$. In the (a)-(c), we show the results for $p=0.05,0.15$ and 0.35 , respectively, and values of degrees of punishment $k=10,50,100$. In the last (d) it is shown the results for $k=10$ and different fractions of negative interactions $p$. Each point is averaged over 100 independent simulations for population size $N=10^{4}$.

\section{Conclusions}

The first numerical attempt to model the fluctuations tax evasion problem was performed by Zaklan et al. [9]. They used Monte Carlo Simulation and the Ising model (equilibrium model) as a tool for the dynamic evolution of Zaklan. In some geometries such as Apollonius and Barabasian networks the Ising model cannot be used due to the lack of phase transition. Lima [12] overcame this obstacle by proposing to use the majority vote model (non-equilibrium model) as a temporal evolution of the Zaklan model. Both proposals were successful in their particular topologies.

Here, we studied the behavior of the tax evasion via the nonequilibrium BCS model. As we saw above the BCS model is a model of opinion formation in a social community. The behavior of the BCS model in relation to control the tax evasion fluctuation is very similar to those found by the Ising and majority vote models. Then, the sociophysics BCS model is another useful tool for studying the tax evasion problem in a community. Therefore, we found the plausible result that tax evasion is diminished by stronger punishment $k$ and audit probability $p_{a}$.

\section{Acknowledgements}

F. W. S. Lima thanks Dietrich Stauffer for many suggestions and fruitful discussions during the development of this work. We 
thank CNPq and FUNCAP for financial support. This work also was supported the system SGI Altix 1350 the computational park CENAPAD.UNICAMP-USP, SP-BRAZIL.

\section{Conflicts of Interest}

The author declares no conflicts of interest regarding the publication of this paper.

\section{References}

[1] Bloomquist, K. (2006) A Comparison of Agent-Based Models of Income Tax Evasion. Social Science Computer Review, 24, 411425. https://doi.org/10.1177/0894439306287021

[2] Föllmer, H. (1974) Random Economies with Many Interacting Agents. Journal of Mathematical Economics, 1, 51-62. https://doi.org/10.1016/0304-4068(74)90035-4

[3] Andreoni, J., Erard, B. and Feinstein, J. (1998) Tax Compliance. Journal of Economic Literature, 36, 818.

[4] Lederman, L. (2003) The Interplay between Norms and Enforcement in Tax Compliance. Public Law Research Paper No. 50. https://doi.org/10.2139/ssrn.391133

[5] Slemrod, J. (2007) Cheating Ourselves: The Economics of Tax Evasion. Journal of Economic Perspective, 21, 25-48. https://doi.org/10.1257/jep.21.1.25

[6] Wintrobe, R. and Gërxhani, K. (2004) Tax Evasion and Trust: A Comparative Analysis. Proceedings of the Annual Meeting of the European Public Choice Society, Berlin, 15-18 April 2004.

[7] Gächter, S. (2006) Conditional Cooperation: Behavioral Regularities from the Lab and the Field and Their Policy Implications. Discussion Papers 2006-03, University of Nottingham, Nottingham.

[8] Frey, B.S. and Togler, B. (2006) Tax Evasion, Black Activities and Deterrence in Germany: An Institutional and Empirical Perspective. IEW-Working Papers 286, Institute for Empirical Research in Economics, University of Zurich, Zurich.

[9] Zaklan, G., Westerhoff, F. and Stauffer, D. (2008) Analysing Tax Evasion Dynamics via the Ising Model. Journal of Economic Interaction and Coordination, $4,1$.

https://doi.org/10.1007/s11403-008-0043-5

[10] Zaklan, G., Lima, F.W.S. and Westerhoff, F. (2008) Controlling Tax Evasion Fluctuations. Physica A: Statistical Mechanics and Its Applications, 387, 5857-5861.

https://doi.org/10.1016/j.physa.2008.06.036

[11] Oliveira, M.J. (1992) Isotropic Majority-Vote Model on a Square Lattice. Journal of Statistical Physics, 66, 273-281. https://doi.org/10.1007/BF01060069 
[12] Lima, F.W.S. (2010) Analysing and Controlling the Tax Evasion Dynamics via Majority-Vote Model. Journal of Physics: Conference Series, 246, Article ID: 012033.

https://doi.org/10.1088/1742-6596/246/1/012033

[13] Lima, F.W.S. (2012) Controlling the Tax Evasion Dynamics via Majority-Vote Model on Various Topologies. Theoretical Economics Letters, 2, 87-93.

https://doi.org/10.4236/tel.2012.21017

[14] Lima, F.W.S. (2012) Tax Evasion and Nonequilibrium Model on Apollonian Networks. International Journal of Modern Physics $C$, 23, Article ID: 1250079 .

https://doi.org/10.1142/S0129183112500799

[15] Lima, F.W.S. (2012) Tax Evasion Dynamics and Zaklan Model on Opinion-Dependent Network. International Journal of Modern Physics C, 23, Article ID: 1250047.

https://doi.org/10.1142/S0129183112500477

[16] Lima, F.W.S. (2015) Tax Evasion Dynamics and Nonequilibrium Zaklan Model with Heterogeneous Agents on Square Lattice. International Journal of Modern Physics C, 26, Article ID: 1550035. https://doi.org/10.1142/S0129183115500357

[17] Lima, F.W.S. (2016) Tax Evasion Dynamics via Non-Equilibrium Model on Directed Small-World Network. Theoretical Economics Letters, 6, 819-826. https://doi.org/10.4236/tel.2016.64086

[18] Crokidakis, N. (2014) A Three-State Kinetic Agent-Based Model to Analyze Tax Evasion Dynamics. Physica A: Statistical Mechanics and Its Applications, 414, 321-328.

https://doi.org/10.1016/j.physa.2014.07.056

[19] Biswas, S., Chatterjee, A. and Sen, P. (2012) Disorder Induced Phase Transition in Kinetic Models of Opinion Dynamics. Physica A: Statistical Mechanics and Its Applications, 391, 3257-3265. https://doi.org/10.1016/j.physa.2012.01.046

[20] Mukherjee, S. and Chatterjee, A. (2016) Disorder-Induced Phase Transition in an Opinion Dynamics Model: Results in Two and Three Dimensions. Physical Review E, 94, Article ID: 062317. https://doi.org/10.1103/PhysRevE.94.062317 